$\begin{array}{lll}\text { ELSEVIER } & \text { Review } & \text { TRENDS in Biotechnology Vol.22 No.2 February } 2004 \\ \text { science@ direct• }\end{array}$

\title{
History, challenges and perspectives of cell microencapsulation
}

\author{
Gorka Orive ${ }^{1}$, Rose Maria Hernández ${ }^{1}$, Alicia Rodríguez Gascón ${ }^{1}$, Riccardo Calafiore ${ }^{2}$, \\ Thomas Ming Swi Chang ${ }^{3}$, Paul de Vos ${ }^{4}$, Gonzalo Hortelano ${ }^{5}$, David Hunkeler ${ }^{6}$, \\ Igor Lacík ${ }^{7}$ and José Luis Pedraz ${ }^{1}$
}

\author{
${ }^{1}$ Laboratory of Pharmacy and Pharmaceutical Technology, Faculty of Pharmacy, University of the Basque Country, \\ Vitoria-Gasteiz, 01006, Spain \\ ${ }^{2}$ Department of Internal Medicine, University of Perugia, Via E. Dal Pozzo, 06126 Perugia, Italy \\ ${ }^{3}$ Artificial Cells and Organs Research Centre, Faculty of Medicine, McGill University, Montreal, Quebec, Canada H3G 1 Y6 \\ ${ }^{4}$ Transplantation Biology and Immunoendocrinology, Department of Pathology and Laboratory Medicine, section of Medical \\ Biology, Groningen, The Netherlands \\ ${ }^{5}$ Department of Pathology and Molecular Medicine, McMaster University, 1200 Main St West, Hamilton, Ontario, Canada L8N 3Z5 \\ ${ }^{6} \mathrm{AQUA}+\mathrm{TECH}$ Specialties SA, Chemin du Chalet-du-Bac CP 28, CH-1283 La Plaine, Geneva, Switzerland \\ ${ }^{7}$ Department of Special Polymers and Biopolymers, Polymer Institute of the Slovak Academy of Sciences, Dubravska cesta 9 , \\ 84236 Bratislava, Slovakia
}

\begin{abstract}
Cell microencapsulation continues to hold significant promise for biotechnology and medicine. The controlled, and continuous, delivery of therapeutic products to the host by immunoisolated cells is a potentially cost-effective method to treat a wide range of diseases. Although there are several issues that need to be addressed, including capsule manufacture, properties and performance, in the past few years, a stepwise analysis on the essential obstacles and limitations has brought the whole technology closer to a realistic proposal for clinical application. This paper summarizes the current situation in the cell encapsulation field and discusses the main events that have occurred along the way.
\end{abstract}

Exciting biotechnological approaches have been developed over the past two decades that have set the stage for tissue and organ replacement, as well as for the continuous and controlled release of therapeutic agents to the host. One of these strategies is cell microencapsulation, which aims to overcome the present difficulties relating to whole organ graft rejection and, consequently, the requirements for the use of immunomodulatory protocols or immunosuppressive drugs. Cell encapsulation, or bioartificial organs, are used to enclose a wide range of bioactive materials within a, generally polymeric, membrane. The latter permits the entry of nutrients and oxygen and the exit of therapeutic protein products. Furthermore, the semipermeable nature of the membrane prevents high molecular weight molecules, antibodies and other immunologic moieties from coming into contact with the encapsulated cells and destroying them as foreign invaders.

One of the earliest demonstrations of transplanted cells in immunoprotective membranes for organ replacement dates back to 1933, when Bisceglie enclosed tumor cells in a polymer membrane and transplanted them into a pig's

Corresponding author: José Luis Pedraz (knppemuj@vc.ehu.es). abdominal cavity [1]. The results showed that the cells survived long enough for it to be concluded that they were not destroyed by the immune system. Thirty years later, Chang introduced the idea of using encapsulation for the immunoprotection of transplanted cells and subsequently evoked the term 'artificial cells' for referring to this concept [2]. This approach was successfully put into practice in the 1970s and 1980s to immobilize xenograft islet cells to aid in glucose control for diabetes in small animal models [3,4]. Since then, tremendous efforts have been made all around the world to advance the understanding of biology, genetics, polymer science and pharmaceutical technology. This has included the development of novel microencapsulation devices and the substitution of the limited supply of primary tissue with more suitable cell lines, improving the long-term delivery of therapeutic peptides either in a local or systemic manner. As a consequence, cell encapsulation has been applied to a wide range of therapeutic treatments including renal failure [5], diabetes [6] and hemophilia [7].

The results achieved from small and large animal models have provided the scientific basis for several clinical trials, including the encapsulation of allogeneic islets for the treatment of diabetes [8], or the most recent of such trials, involving the immobilization of cytochrome P450 enzyme expressing cells for the eradication of pancreatic cancer [9]. However, recent progress in this multidisciplinary field of research is lagging behind the expectations expressed only a few years ago $[10,11]$. One of the main reasons for this is that, although the leading groups working in this field have excellent tissue and biocompatible materials, the overall success rate is relatively limited owing to either lack of, or uncertainty in, reproducibility. As a consequence, many essential questions remained unanswered, and this has hampered progress. In the past six years, however, the strategy in the field has changed, applying a stepwise analysis to the 
essential research questions instead of an approach via trial and error. Not surprisingly, this has renewed the excitement and hopes surrounding this cell-based technology.

\section{Potential advantages}

In light of the increasing incidence of age-related diseases and the current desperate shortage of donor organs, the hope that encapsulated cells might be used as a therapeutic tool is increasingly being realized. Furthermore, the potential of this approach includes encapsulated cells which supply the host with regulated and/or continuous 'de novo' delivery of therapeutic product. These artificial cells can be transplanted into a variety of tissues and organs, making the technology suitable for local (solid tumors), regional (brain), oral or systemic (intraperitoneal) delivery of therapeutics. When long-term continuous delivery of therapeutic agents is required, the cost of encapsulated cells can be off-set by the cost of the therapeutic product. Moreover, the chemical composition of the capsule can be the same for treating a large number of patients, regardless of their human leukocyte antigen (HLA) class, making this approach cost-effective. Certainly, if the pharmacoeconomics of the life cycle of cell therapy are favorable, compared with either existing whole organ transplantation or a lifetime of exogenous hormone delivery, the insurance companies will reduce the payments to hospitals for traditional therapies and increase the demand for cell transplantation. Nevertheless, biotechnology firms, whether startups or multinationals, are faced with billion dollar development programs and a decade of strongly negative cash flows, which put at risk the viability of both the project and the company itself, or, as has recently been seen with companies such as Advanced Tissue Science and Cytotherapeutics.

The authors believe that cell microencapsulation has enormous potential to make significant contributions to medicine over the next decade. However, to be a viable complement to the new modes of cell transplantation therapy $[12,13]$, encapsulation technology has to fulfill the strict requirements applicable to these types of therapeutic strategies, i.e. performance, biosafety, biocompatibility, retrievability, stability, availability, purity, characterization and cost.

\section{Materials}

It is widely understood that biocompatible materials, which do not interfere with cell homeostasis, have to be applied within capsules to allow for survival of the enclosed cells, and that the polymers used for allo- and xenotransplantation will differ, with the latter process probably requiring a much tighter membrane. Furthermore, it is essential that the capsules should have an adequate mechanical stability to allow for exchange of nutrients and metabolic waste.

In the search for a better microencapsulation design, many types of natural and synthetic polymers are being explored. Finding a suitable material to form a capsule is challenging. The overwhelming majority of the literature has employed sodium alginate (composed of mannuronic and guluronic $(\mathrm{G})$ dimers) as the polyanion and poly-L-lysine (PLL) as the polycation for microcapsule formation. The former is probably the most suitable material for capsule design, in part because of its excellent biocompatibility and biodegradability, although the biocompatibility of the alginates in relation to their composition is a matter of debate. Whereas some researchers believe that high $\mathrm{G}$ acid alginates are the most suitable types, others prefer alginates with intermediate or low $G$ content [14]. All of this controversy is caused by the lack of a standard definition for high $G$ alginate and the difference in the geometry of the capsules employed in the various experimental studies. Very recently, the group in Trondheim developed an enzymatically modified alginate, improving the biocompatibility of sodium alginate/poly-Llysine microcapsules [15].

Similarly, to prevent foreign body reactions, the cation content (e.g. PLL) should be kept to a minimum, or be neutralized with an outer purified alginate coating, because this polycation attracts inflammatory cells, thereby inducing necrosis of the encapsulated cells [16]. Indeed, some authors go as far as to recommend uncoated beads for allotransplantation [17], recognizing that the polycation needed in xenografts will be insufficient to block the direct and indirect immune activation pathways. Although much

Table 1. Alternative encapsulation systems to the classical alginate-poly-L-lysine-alginate microcapsules

\begin{tabular}{|lll|}
\hline Microcapsule design & Advantages \\
\hline Alg $^{\text {a }}$-PLO & Refs \\
Alg-Cellulose sulfate-PMCG & Increased biocompatibility \\
HEMA-MMA & Independent adjustment of capsule parameters \\
Agarose-polystyrene sulfonate & Improved mass transfer, stability and durability \\
Alg-Agarose & Blocks the activation of complement via enhancing the activity of C1 inhibitors & [25] \\
Barium-alg & Increased mechanical stability & [37] \\
PDADMAC & Increased mechanical and chemical stability \\
Alg-chitosan & Increased mechanical stability & [39] \\
PVA & Increased biocompatibility \\
Photopolymerised PEG & [40] \\
\hline
\end{tabular}

${ }^{a}$ Alg, alginate;

bPLO, poly-L-ornithine;

${ }^{\mathrm{C}} \mathrm{PMCG}$, poly (methylene-co-guanidine);

dHEMA-MMA, hydroxyethyl methacrylate-methyl methacrylate;

ePDADMAC, polydiallyldimethyl ammonium chloride;

fPVA, polyvinylalcohol;

gPEG, poly (ethylene glycol). 
effort has been focused on identifying alternative systems to alginate/PLL chemistry (Table 1), none have overcome all of the disadvantages of the PLL. Therefore, although the polycation is required for xenotransplantation, and this renders the solution problematic, the allografts are likely to revert to the uncoated alginate beads [18].

\section{Diffusion and mass transport}

The premise in designing a device with a semipermeable membrane is to adjust its permeability, in terms of the entry and exit of molecules. The appropriateness of a membrane depends on the control it allows over both the size-based exclusion and rate of diffusion of the molecules which either should or should not permeate the membrane to control the survival, as well as the metabolic efficacy, of the graft. Generally, the process of transporting various species across a membrane, characterized as the membrane permeability, is governed by both the thermodynamic parameter known as the equilibrium partition coefficient and the kinetic parameter known as the diffusion coefficient. Comprehensive reviews devoted to the issue of permeability in cell encapsulation devices have recently been published [19,20].

The type and size of a solute, interactions between the solute and the membrane, and the membrane thickness are the chief factors determining the rate of diffusion, as well as the partitioning, of the solute and, thereby, the permeation properties. Furthermore, efficient diffusion and a minimal delay in the exchange of the substances across the membrane are crucial for influencing the membrane environment and proximity to the circulation [21].

The development of currently used materials has mostly been focused on setting the permeability parameters and testing them in vitro. However, the most sensible way of optimizing the technique of encapsulating cells should involve assessing the diffusion and permeability properties in vivo. This has been done by testing an implanted diffusion chamber [22] in vivo and also the permeability of an explant [23]. The proposed metrics for the characterization of immunoisolation devices include this step as crucial for standardization prior to being submitted to the Food and Drug Administration (FDA) [24].

\section{Microcapsule mechanical stability}

The initial enthusiasm regarding the alginate-PLL microcapsule was dampened after realizing that the membrane exhibits poor mechanical stability. The additional efforts in achieving the improved mechanical stability and durability of capsules resulted in a modification to the chemical composition of the membrane (Table 1). One approach involved replacing PLL with poly-L-ornithine (PLO) and decreasing capsule size from 800 to $400 \mu \mathrm{m}$ [25]. In another study, polyanions with both weak (alginate) and strong (cellulose sulfate) interactions with the polycation poly (methylene-co-guanidine) provided high and controlled mechanical strength and capsule durability [26]. The importance of having both strong and weak interactions controlling mechanical and chemical stability has also been adopted in the recently patented polyanionic material, Biodritin, formed by covalently bound chondroitin sulfate and sodium alginate [27].
Other approaches have involved alginate beads made from crosslinked barium ions, as used for allograft islet transplantation in non-obese diabetic (NOD) mice [17], optimization of the alginate-poly (methylene-co-guanidine)alginate microcapsules [28], coating the alginate beads with alternating layers of different polyanions and polycations, and even the incorporation of an inorganic silicabased polycation, which was successfully tested with rat islets in vitro and as empty capsules in vivo [29].

\section{Rejection pathways}

Several pathways are involved in the rejection of immunoisolated cells. In allograft immunity, because immune mechanisms mainly consist of direct engagement of T lymphocyte sub-populations (i.e. $\mathrm{CD}^{+}$) with donor cells, the physical isolation provided by the capsules should prevent cell-to-cell contact between the encapsulated tissue and the host's immune system, thereby facilitating the immunological acceptance of the graft. In the case of xenograft immunity, microcapsules could prevent access to antibodies as well as complement fractions, because of the membrane's nominal molecular weight cut-off (MWCO) selection properties. However, the risk for activation of $\mathrm{CD}^{+}$-driven cellular reactivity cannot definitively be ruled out. In fact, small molecules ( $\sim \mathrm{a}$ few $\mathrm{kD}$ ), or 'shed antigens', such as proteins or enzymes, could easily cross the capsular membrane, triggering a significant inflammatory cell reaction. In this scenario, small cytokines, nitric oxide and free oxygen radicals released from the inflammatory cells, individually or collectively, could severely harm, and possibly destroy, the encapsulated cells. Finally, the recurrent autoimmunity should mimic the cellular arm of the xenograft-directed immune response, and then affect the transplanted cells only by the extent of the inflammatory cell tissue response that is generated in the immediate vicinity of the encapsulated cells the graft.

To address the problem of graft rejection, it is essential to select highly purified and biocompatible polymers. Specifically, a reduction in endotoxin content and the elimination of proteins are essential. Furthermore, live cells and their secretory products could play a role in the biological acceptance of the encapsulated cells, as well as the graft site and the adopted transplant procedure. Spherical shape and suitable size of the microcapsule are also major challenges for optimal immunocompatibility. The larger the volume of the grafted encapsulated tissue, the more frequent the incidence of adverse side effects in the recipients. Finally, it is still possible to incorporate either chemical agents (i.e. vitamins D3 and E) or cellular systems (i.e. Sertoli cells) that can act as antioxidizing and immunomodulatory factors, within a multicompartmental microcapsule prototype [30]; this should significantly strengthen the immunobarrier capacity, although the crucial role of all of these agents still remains to be proven.

\section{Cell lines}

Judicious choice of encapsulated cells is essential for the success of any biomedical application. Over the past few years, many different cell sources have been immobilized 


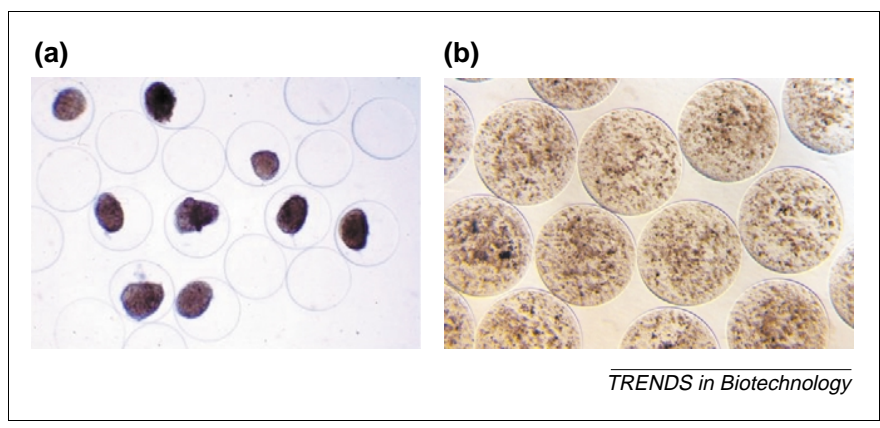

Figure 1. Different cell sources have been employed in cell encapsulation technology, ranging from the islets of Langerhans (a), the most commonly used cell type, to novel approaches using encapsulated stem cells and hepatocytes (b).

(immunoisolation is a specific form of a more general term - immobilization - with the former referring only to transplantation) (Figure 1), although clearly not all cells are suitable for encapsulation. Several factors should be carefully considered when choosing appropriate cell types for immobilization. The use of allogeneic versus xenogeneic cells has important immunological repercussions. Furthermore, cells that proliferate following encapsulation could eventually fill the entire capsular space and lead to diminished efficacy of therapeutic diffusion. As a result, the long-term viability of encapsulated cells is often compromised [31]. By contrast, cells that do not proliferate after encapsulation, such as myoblasts, have the potential to deliver therapeutic products for longer periods of time. Finally, genetic engineering has certainly increased the success of cell microencapsulation for biomedical applications. In essence, genes can now be used as templates, cells as reactors to secrete the final product and capsules as immunoisolation vehicles for drug delivery in vivo. However, the use of genetically modified cells will have to be balanced with respect to safety and stability of gene expression. Some of the cell sources employed for cell immobilization purposes are reviewed in Table 2.

\section{Applications}

The economic costs for standard medical treatment have contributed to the re-evaluation of the affordability of public and private healthcare options in many countries. In the USA alone, hundreds of billion dollars, $\sim 10 \%$ of the Gross National Product, are spent on the treatment of neurodegenerative and endocrine diseases and inborn errors of metabolism, as well as a variety of acute and chronic organ failures. Eighty five percent of these expenses are a result of the morbidity associated with these diseases. For diabetes alone, the incidence of which increase by $3-4 \%$ per year, the annual direct and indirect costs exceed 90 billion dollars (http://www.jdf.org). Given the potential of cell microencapsulation technology to treat these diseases, the societal and pharmacoeconomic impact of such devices would be important.

Cell encapsulation has, thus far, focused on creating a bioartificial pancreas by encapsulating pancreatic islets both allografts and xenografts. Pancreatic islet xenografting has prompted major concern because of the possible transmission of infectious agents, particularly the porcine endogenous retrovirus (PERV), from the donor to the recipient [32]. Despite recent results suggesting that encapsulated xenogeneic islets are less capable of binding and taking up human cytokines [33], the risks involved in xenotransplantation and the long developmental and regulatory pipelines, publicly funded allotransplantation seems more likely to succeed than privately supported xenotransplantation.

However, the exciting improvements in outcomes following clinical islet transplantation using the 'Edmonton protocol' have renewed hope for patients with type 1 diabetes [12]. Although excellent results have been obtained, researchers believe that alternative strategies will be required to make the treatment safer and more available for these patients. These strategies could include developing the means to minimize or totally avoid all immunosuppression, hopefully through cellular encapsulation or other strategies to induce robust tolerance, combined with alternative cell sources including stem cell or xeno-derived insulin secreting cells. Yet another important challenge is represented by the banking of enough donor tissues. Ultimately, cell source development will have to focus on allografts of immortalized cells,

Table 2. Different cell sources employed for cell immobilization purposes

\begin{tabular}{|c|c|c|}
\hline Cell type & Application & Material \\
\hline Fibroblasts & Metabolic deficiencies, neurotrophic factors, epilepsy & Alginate, HEMA-MMA ${ }^{a}$ \\
\hline Myoblasts & Metabolic deficiencies, neurotrophic factors, cancer & Alginate, HEMA-MMA \\
\hline Kidney cells & Hemophilia, neurotrophic factors, antiangiogenesis & Alginate \\
\hline Pancreatic islets & Diabetes & $\begin{array}{l}\text { Alginate, Agarose-polystyrene/sulfonic acid, } \\
\text { AN69, Cellulose sulfate }\end{array}$ \\
\hline Ovary cells & Fabry disease & Alginate, HEMA-MMA \\
\hline Parathyroid cells & Artificial organs & Alginate \\
\hline Hepatocytes & Liver transplantation & Alginate, HEMA-MMA \\
\hline Chondrocytes & Bone and cartilage regeneration & Alginate \\
\hline Leydig cells & Hormone replacement & Alginate \\
\hline Adrenal chromaffin cells & Parkinson's disease, chronic pain & Alginate \\
\hline Stem cells & Bone regeneration & Alginate \\
\hline PC12 pheochromocytoma cells & Neurotrophic factors, neurotransmitters & Alginate, HEMA-MMA \\
\hline Myeloma cells & Hepatic growth factor & Alginate \\
\hline Hybridoma cells & Antibody production & Alginate, acetate, alginate-agarose \\
\hline Tumor cells & Cancer vaccine, interleukins & Alginate, chitosan \\
\hline Virus producer cells & Cancer & Cellulose sulfate \\
\hline Bacteria & Elimination of urea & Alginate \\
\hline
\end{tabular}

${ }^{a}$ HEMA-MMA, hydroxyethyl methacrylate-methyl methacrylate. 
although this will be complicated by the fact that cotransplantation of cells will be required. Recently, pilot phase-1 clinical trials of alginate/PLO microencapsulated human islet grafts in nonimmunosuppressed patients with type 1 diabetes have been recently approved (September 2003) by the Italian Ministry of Health, to be conducted at the University of Perugia, Italy (personnal communication by R. Calafiore).

Other successful studies employing immobilized primary cells include the extracorporeal immunoisolation of hepatocytes and the macroencapsulation of adrenal cells for the treatment of chronic pain and the allotransplantation of microencapsulated parathyroid tissue [34].

Cell microencapsulation also has implications for gene therapy, in that cells can be genetically engineered to secrete a desired genetic product prior to their encapsulation. For example, Löhr et al. [35] administered microencapsulated cells transfected with the CYP2B1 gene into a tumor, with the aim of creating a therapeutic vehicle that could target delivery of a cytotoxic drug specifically to the site of the tumor. The results showed that patients tolerated the treatment without any side effects and that the median survival of the patients was the same as that of the control group. Encapsulated cells are also being explored as a therapeutic tool for an in vivo gene therapy approach. To avoid the repeated delivery of a vector in vivo, and thereby avoid the development of an immune response against the vector, retroviral packing cells that continuously produce recombinant retroviral vector particles were encapsulated in beads made from cellulose sulfate and polydiallyldimethyl ammonium chloride. Encapsulated virus-secreting cells remained viable and released virus into the culture medium for at least six weeks, both in vitro and in vivo [36].

\section{Future perspectives}

Microencapsulation has a potentially significant future in medicine and biotechnology, the latter including agricultural and environmental applications. However, several significant challenges still face cell microencapsulation technology. For example, dosing should be carefully controlled by using cells that thrive and proliferate to a limited degree only, thereby preventing uncontrolled cell growth and overcrowding of the cells within the immunoisolation device. Moreover, constituent polymers should be standardized in terms of physicochemical properties/ composition, purity grade, reaction conditions and source reproducibility, so as to fulfill biosafety and efficacy requirements uniformly and reduce the variability in the results achieved by different laboratories [10]. Indeed, transplant-grade biomaterials, which are characterized by their batch numbers, are required, in the same way as in the case of enzymes used, for example, in tissue digestion.

Another challenge is the scaling-up of the manufacturing process while maintaining a controlled environment, operational discipline and rigorous quality controls. This step will be essential for allowing cell microencapsulation technology to enter human clinical trials and thereby become a real clinical therapeutic strategy. Another major area of study is the retrievability associated with the microcapsules and the banking and culture of suitable cell sources. The importance of protocol design in yielding donor cells of adequate quantity and quality is paramount for the success of this technology.

The next step will be likely to involve the development of 'super-specialized' microcapsules that embody multiple micro-compartments, each one dynamically interacting with the other, containing cells or pharmacological agents that interact favorably with the 'primary' cellular product. Such capsules will not be more complex, chemically or biologically, but will be based on simple engineered materials, assembled in the correct manner. The final aim is to continuously improve cell life expectancy and functional performance, and eventually to establish a selfperpetuating cell regeneration pattern, to address the treatment of high social impact diseases, such as diabetes, Parkinson's disease or Alzheimer's disease.

\section{References}

1 Bisceglie, V. (1933) Uber die antineoplastische immunitat; heterologe Einpflnzung von Tumoren in Huhner-embryonen. Ztschr. Krebsforsch 40, $122-140$

2 Chang, T.M.S. (1964) Semipermeable microcapsules. Science 146 , $524-525$

3 Chick, W.L. et al. (1975) Beta cell culture on synthetic capillaries: an artificial endocrine pancreas. Science 187, 847-848

4 Lim, F. and Sun, A.M. (1980) Microencapsulated islets as bioartificial endocrine pancreas. Science 210, 908-909

5 Prakash, S. and Chang, T.M.S. (1996) Microencapsulated genetically engineered live $E$. coli DH5 cells administered orally to maintain normal plasma urea level in uremic rats. Nat. Med. 2, 883-887

6 Sun, Y.L. et al. (1996) Normalization of diabetes in spontaneously diabetic cynomologous monkeys by xenografts of microencapsulated porcine islets without immunosuppression. J. Clin. Invest. 98, 1417-1422

7 Hortelano, G. et al. (1996) Delivery of human factor IX in mice by encapsulated recombinant myoblasts: a novel approach towards allogeneic gene therapy of hemophilia B. Blood 87, 5095-5103

8 Soon-Shiong, P. et al. (1994) Insulin independence in a type 1 diabetic patient after encapsulated islet transplantation. Lancet 343, 950-951

9 Löhr, M. et al. (2001) Microencapsulated cell-mediated treatment of inoperable pancreatic carcinoma. Lancet 357, 1591-1592

10 Orive, G. et al. (2003) Cell encapsulation: promise and progress. Nat. Med. 9, 104-107

11 Lanza, R.P. et al. (1996) Encapsulated cell technology. Nat. Biotechnol. 14, 1107-1111

12 Shapiro, A.M.J. et al. (2000) Islet transplantation in seven patients with Type 1 diabetes mellitus using a glucocorticoid-free immunosuppressive regimen. N. Engl. J. Med. 343, 230-238

13 Lanza, R.P. et al. (1999) Human therapeutic cloning. Nat. Med. 5, 975-977

14 Klöck, G. et al. (1997) Biocompatibility of mannuronic acid-rich alginates. Biomaterials 18, 707-713

15 King, A. et al. (2003) Improvement of the biocompatibility of alginate/ poly-L-lysine/alginate microcapsules by the use of epimerized alginate as a coating. J. Biomed. Mater. Res. 64, 533-539

16 Strand, B.L. et al. (2001) Poly-L-lysine induces fibrosis on alginate microcapsules via the induction of cytokines. Cell Transplant. 10, $263-275$

17 Duvivier-Kali, V.F. et al. (2001) Complete protection of islets against allorejection and autoimmunity by a simple barium-alginate membrane. Diabetes 50, 1698-1705

18 Thu, B. et al. (1996) Alginate polycation microcapsules. II. Some functional properties. Biomaterials 17, 1069-1079

19 Schuldt, U. and Hunkeler, D. (2000) Characterization methods for microcapsules. Minerva Biotec. 12, 249-264

20 Uludag, H. et al. (2000) Technology of mammalian cell encapsulation. Adv. Drug Deliv. Rev. 42, 29-64

21 De Vos, P. et al. (2002) Considerations for successful transplantation of encapsulated pancreatic islets. Diabetologia 45, 159-173

22 Rafael, E. et al. (1999) In vivo evaluation of glucose permeability of an 
immunoisolation device intended for islet transplantation: a novel application of the microdialysis technique. Cell Transplant. 8, $317-326$

23 Sharkawy, A.A. et al. (1997) Engineering the tissue which encapsulates subcutaneous implants. Diffusion properties. J. Biomed. Mater. Res. 37, 401-412

24 Hunkeler, D. et al. (2001) Objectively assessing bioartificial organs. Ann. N. Y. Acad. Sci. 944, 456-471

25 Calafiore, R. et al. (1999) Transplantation of minimal volume microcapsules in diabetic high mammalians. Ann. N. Y. Acad. Sci. $875,219-232$

26 Wang, T. et al. (1997) An encapsulation system for the immunoisolation of pancreatic islets. Nat. Biotechnol. 15, 358-362

27 Mares-Guia, M. and Ricordi, C. Hetero-polysaccharide conjugate and methods of making and using the same. US Patent 6,281,341

28 Orive, G. et al. (2003) Development and optimisation of alginatePMCG-alginate microcapsules for cell immobilisation. Int. J. Pharm. $259,57-68$

29 Sakai, S. et al. (2001) Synthesis and transport characterization of alginate/aminopropyl-silicate/alginate microcapsule: application to bioartificial pancreas. Biomaterials 22, 2827-2834

30 Luca, G. et al. (2003) Multifunctional microcapsules for pancreatic islet cell entrapment: design, preparation and in vitro characterization. Biomaterials 24, 31091-31114

31 Chang, P.L. and Bowie, K.M.M. (1998) Development of engineered cells for implantation in gene therapy. Adv. Drug Deliv. Rev. 33, 31-43

32 Murphy, F.A. (1996) The public health risk of animal organ and tissue transplantation into humans. Science 273, 746-747

33 De Vos, P. and Marchetti, P. (2002) Encapsulation of pancreatic islets for transplantation in diabetes: the untouchable islets. Trends Mol. Med. 8, 363-366
34 Hasse, C. et al. (1997) Parathyroid allotransplantation without immunosuppression. Lancet 350, 1296-1297

35 Löhr, M. et al. (2002) Microencapsulated, CYP2B1-transfected cells activating ifosfamide at the site of the tumor: the magic bullets of the 21st century. Cancer Chemother. Pharmacol. 49 (Suppl. 1), S21-S24

36 Saller, R.M. et al. (2002) Encapsulated cells producing retroviral vectors for in vivo gene transfer. J. Gene Med. 4, 150-160

37 Tse, M. et al. (1996) Secretion of recombinant proteins from hydroxyethyl methacrylate-methyl methacrylate capsules. Biotechnol. Bioeng. 51, 271-280

38 Iwata, H. et al. (1994) Strategy for developing microbeads applicable to islet xenotransplantation into a spontaneous diabetic NOD mouse. J. Biomed. Mater. Res. 28, 1201-1207

39 Orive, G. et al. (2001) Microencapsulation of an anti VE-cadherin antibody secreting 1B5 hybridoma cells. Biotechnol. Bioeng. 76, $285-294$

40 Zekorn, T. et al. (1992) Barium-alginate beads for immunoisolated transplantation of islets of Langerhans. Transplant. Proc. 24, 937-939

41 Dautzenberg, H. et al. (1999) Development of cellulose sulfate-based polyelectrolyte complex microcapsules for medical applications. Ann. N. Y. Acad. Sci. 875, 46-63

42 Bartkowiak, A. and Hunkeler, D. (1999) New microcapsules based on oligoelectrolyte complexation. Ann. N. Y. Acad. Sci. 875, 36-45

43 Iwata, H. et al. (1990) The use of photocrosslinkable polyvinyl alcohol in the immunoisolation of pancreatic islets. Transplant. Proc. 22, 797-799

44 Cruise, G.M. et al. (1999) In vitro and in vivo performance of porcine islets encapsulated in interfacially photopolymerized poly(ethylene glycol) diacrylate membranes. Cell Transplant. 8, 293-306

\section{TIBTEC - making the most of your personal subscription}

High-quality printouts (from PDF files)

Links to other articles, other journals and cited software and databases

$$
\text { All you have to do is: }
$$

Obtain your subscription key from the address label of your print subscription.

Then go to http://www.trends.com, click on the Claim online access button and select

$$
\text { Trends in Biotechnology. }
$$

You will see a BioMedNet login screen.

Enter your BioMedNet username and password. If you are not already a BioMedNet member, please click on the Register button. Once registered, you will be asked to enter your subscription key. Following confirmation, you will have full access to

\section{Trends in Biotechnology.}

If you obtain an error message please contact Customer Services (info@current-trends.com) stating your subscription key and BioMedNet username and password. Please note that you only need to enter your subscription key once; BioMedNet 'remembers' your subscription. Institutional online access is available at a premium. If your institute is interested in subscribing online, please ask them to contact reviews.subscribe@ biomednet.com 